\title{
ELECTROCHEMICAL CELLS AS ELECTRICAL CIRCUIT ELEMENTS
}

\section{B. BREYER}

Department of Pharmacology, University of Milan (Italy)

H. H. BAUER

Department of Chemistiy, University of Michigan, Anu Arbor (U.S.A.)

Dedicated to Professor Dr. MI. Von StackelserG on his 7oth birthday

(Received April I5th, Ig66)

The bchavior of electrochemical cells when subjected to alternating electrical signals has been studied since the latter part of the Igth century (for a short review, see BREYER AND BAUER ${ }^{1}$ *. Our understanding of the processes involved remains far from complete (e.g., the properties of solid electrodes with respect to frequency dispersion) but it does seem to be generally true that a cell in which no faradaic process occurs (i.e., where there is no electron transfer across electrode-solution boundaries) can be satisfactorily represented electrically by series (on occasion, also parallel) combinations of capacitors and resistors over wide ranges of frequencies; on the other hand, a cell in which a faradaic process is taking place cannot be represented by any combination of conventional circuit elements ${ }^{2}$, whether passive (resistors, capacitors, inductors) or active (vacuum tubes, transistors).

The electrolytic capacitor is an example of the use, as a practical circuit element, of a sort of electrochemical cell in which no faradaic process occurs; for many years, these electrolytics have been the only practical capacitors of high capacity obtainable in a useful physical size and at low cost.

It is the purpose of this discussion to bring under consideration the possible merits, as circuit elements, of electrochemical cells in which faradaic processes do, or can be made to, occur; such elements may logically and conveniently be referred to as faradaics.

\section{PROPERTIES OF FARADAICS}

The fact that faradaics behave in a way that cannot be simulated by combining the conventional circuit elements, has, as one corollary, that the properties of faradaics when exposed to alternating signals differ in novel ways from the properties exhibited under non-periodic conditions. To take as examples the most widely known experimental situation, we may compare the form of a conventional polarogram and those of a.c.1 and second-harmonic ${ }^{3,4}$ a.c. polarograms (Fig. I). Under the influence of a direct voltage (bias) only, a faradaic typically displays a high resistance but with a

- Throughout this article, no specific references will be given for statements regarding observations or theories that have become generally accepted by workers in the field of alternating current polarography. Substantiation and details in such instances will be found in ref. $I$. 
minimum at one particular bias, the value of this minimum resistance being determined by the electrochemical system used. In the presence of a small alternating voltage superposed on the bias voltage, a faradaic behaves as an electrolytic at bias voltages where no electrochemical reaction occurs, where electron transfer does take place, however, the impedance is lowered-it may be very drastically - to a value that is quite different from that of the minimum resistance to direct current shown at the same bias; the phase angle between the alternating current and the alternating voltage is determined by the nature of the electrochemical reaction occurring and the frequency of the alternating signals, and may take any value between zero and $x / 2$; rectification effects are observed (a direct component and higher harmonics are generated-vide the second-harmonic polarogram); and the impedance of the faradaic may be negatives-7.

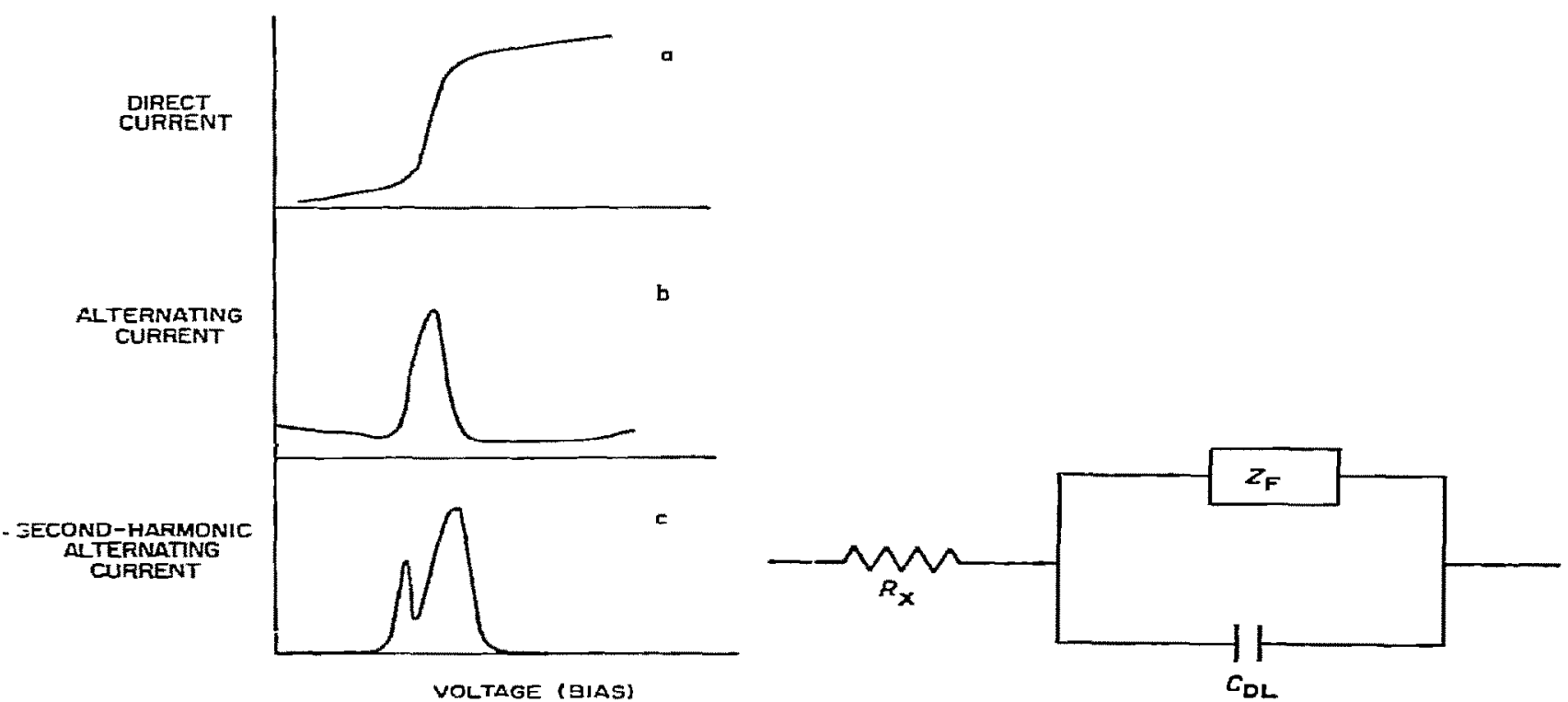

Fig. I. Polarograms illustrating electrical properties of faradaics.

Fig. 2. Electrical equivalent of electrochemical cell.

Figure I represents schematically the characteristic of one sort of faradaic. Much information about particular values obtainable, the effects of parameters such as amplitude and frequency of the alternating signal, and so on, can be gleaned from the literature devoted to the study of faradaic processes by means of alternating signals. In this literature, the electrochemical cell is usually represented in the way shown in Fig. 2, where $Z_{F}$ is the element representing the electrochemical reaction, $C_{D x}$ is the (almost purery) capacitive element due to the electrical double-layer at the interphase boundary, and $R_{x}$ represents ohmic effects of electrolyte and electrodes. Faradaics behave as the whole circuit of Fig. 2, unless faradaic processes at more than one electrode are important, when elaboration would be necessary. This point is raised to make clear the considerations involved in the design of faradaics: at least three (largely) independent factors $\left(R_{\mathbf{X}}, C_{\mathbf{D L}}, Z_{F}\right)$ need to be taken into consideration. 


\section{Ohmic resistance}

The magnitude of $R_{\mathbf{X}}$ depends on cell geometry and on the nature and activity of the electrolytes present: it may be so small as to be negligible for many purposes (say Io $\Omega$ ) and merely modifies whatever novel properties the faradaic may exhibit as a result of the nature of $Z_{\mathrm{F}}$.

\section{Double-layer capacity}

The magnitude of $C_{\mathrm{DL}}$ also depends on cell geometry (in particular, size of the electrodes) and on the electrolytes present. The capacity/unit area of electrode is often of the order of $20 \mu \mathrm{F} / \mathrm{cm}^{2}$ in aqueous solutions, but it is affected by the bias voltage and may be very sensitive to the presence of surfactants that need not themselves be electrolytes. There are three main points worth mentioning here:

(a) The effect of surfactants in even small amount on the magnitude of $C_{D L}$ (and, even more, on that of $Z_{F}$ ) makes important the control of purity of reagents when preparing faradaics.

(b) In the presence of surfactants, $C_{\mathrm{DL}}$ may vary with the bias to a remarkable extent (tensammetric waves ${ }^{8}$ and pseudo-capacity peaks ${ }^{9}$ associated with adsorptiondesorption potentials). At some bias voltages, the electrical characteristics of cells in which adsorption-desorption, but not electron transfer, is occurring can be quite as unusual as with faradaics. Thus, another class of electrochemical circuit elements can be envisioned (tensammetrics). With these, an even greater discrepancy between resistance to direct current (high) and impedance to altcrnating current (low) is observed than with faradaics; phase relationships are different (e.g., HAYES AND BAUER ${ }^{10}$ ); rectification effects are also observed 11 .

(c) Often, the magnitude of $C_{\mathrm{DL}}$ can be made sufficiently small that it does not greatly shunt $Z_{F}$ and therefore need not greatly influence the behavior of the faradaic.

\section{Electrochemical reaction- $Z_{F}$}

(a) Magnitude of the impedance. Usually, the magnitude of the impedance, $Z_{F}$, decreases in inverse proportion to the concentration of the electroactive species involved. It may be as low as a few ohms or even less, or so great that $C_{D \mathrm{DI}}$ becomes the determining impedance for the whole cell. In some systems the impedance can be a non-linear function ${ }^{12,13}$ of the concentration of the electroactive species.

The impedance decreases with increasing frequency, as $\omega^{-\frac{1}{3}}$ at lower frequencies and less rapidly at higher frequencies. The impedance is independent of amplitude of the signal at small amplitudes, but increases appreciably with amplitudes of tens of millivolts or more 14,15 .

(b) Phase shift. With many systems, the phase shift is $\pi / 4$ at low frequencies and decreases to zero as the frequency increases. Exceptions have been ascribed to particular conditions at the surface of the electrode (adsorption ${ }^{16}$, influence of doublelayer structure ${ }^{17}$ ), which may in fact produce a negative-impedance effect ${ }^{18}$.

(c) Rectification. $Z_{F}$ displays rectifying properties: an alternating signal produces a direct component and higher harmonics. These effects are enormously influenced by the bias voltage (e.g., Fig. Ic) and also depend on the nature of the system and on the frequency and amplitude of the signal. This type of rectification is fundamentally different from that observed with vacuum tubes and solid-state

$$
\text { J. Electroanal. Chem, I2 (I966) 4II-4I5 }
$$


devices, particularly in that it is of low efficiency-which might in some cases be advantageous.

\section{UTIIITY OF FARADAICS AND TENSAMMETRICS}

Electrochemical cells have electrical properties that are novel and, consequently, circuits employing them as elements display unique behaviour. It would be foolish to attempt to forecast the whole range of fossible applications of these elements, beyond pointing out-as above - some of the electrical characteristics that make them unique, and indicating (see below) some of the most obvious instances where faradaics offer possibilities that seem to be unique or unusually convenient. It is the authors' main hope and intention, to interest in this field workers who are creatively concerned with electrical circuitry, by drawing attention to these possibilities and to the literature which contains detailed studies of the behavior of the proposed elements under a wide variety of conditions.

If sufficiently interesting uses are found, practical considerations-purity of chemicals, stability under various environments, physical ruggedness and size-will not offer serious obstacles to the incorporation of faradaics and tensammetrics in electrical instrumentation.

Some possible applications include:

\section{(I) Constant-phase-shift elements}

With an electrochemical process that is sufficiently fast, the phase shift is $\pi / 4$, independent of frequency over a range of frequencies. This is characteristic of what GRAHAME termed ${ }^{2}$ the Warburg impedance, an electrochemical reaction limited by diffusion of the electroactive species. Combination of a Warburg impedance and a resistor produces elements having frequency-independent phase shifts between $\pi / 4$ and zero.

\section{(2) Negative-impedance elements}

$Z_{\text {F }}$ can display behavior indicative of a negative impedance. Moreover, this phenomenon is probably explicable on the basis of contemporary theory ${ }^{2.18}$ so that the design of faradaics with specific negative-impedance properties can be attempted.

A negative-impedance element is also, apparently, the first type of faradaic to have been used in a practical electrical circuit: TAMAMUSHI has shown that a circuit containing a negative-impedance faradaic, an inductor, and a source of direct voltage can produce undamped sinusoidal signals. As pointed out by TAMAMUSHI, this is an electrochemical oscillator that converts direct voltage into an alternating signal and is also- of great interest as a new way of studying faradaic processes, with potential applications in biophysics.

\section{(3) Voltage standards}

Electrochemical cells have long been used as sources of voltage of standard magnitude. Use of the rectifying properties of faradaic processes might sometimes be useful in this connection. Thus, the bias voltage at which the higher harmonics vanish can in some cases be located to a.small fraction of a millivolt, and this accuracy in no way depends on the magnitude of the bias voltage. 


\section{ACKNOWLEDGEMENTS}

The authors are indebted to their friends and colleagues for helpful discussions of a number of relevant points. It is a pleasure to mention particularly the patient and helpful advice of L. MEDINa, and the enthusiasm of S. KEEN who envisaged multielectrode electrolytic analogues of various solid-state devices.

One of us (H.H.B.) wishes to acknowledge the hospitality and stimulation afforded him by Professor Philip J. Elving at the University of Michigan, and facilities and financial support from the U.S. Atomic Energy Commission and the Institute of Science and Technology at the University of Michigan.

\section{REFERENCES}

I B. BREYeR AND H. H. BAUER, Alternating Current Polarography and Tensammetry, Joh Wiley/ Interscience, New York, I963.

2 D C. Grahiare, J. Electrochem. Soc., 99 (I952) $370 \mathrm{C}$.

3 H. H. Bauer, J. Sci. Ind. Res. (India), 24 (1965) 372.

4 H. H. Bauer aNd D. C. S. Foo, Australian J. Chem., in press.

5 H. H. Bauer, D. L Smith aNd P. J. ElviNG, J. Ami. Cheml. Soc, 82 (I960) 2094.

6 N. Tanaka and R. Tamamushi, Proceedings ist Australian Confegence Electrochemist'y, Pergamon Press, Oxford, 1964, p. 248.

7 R. Tamanisui, $J$. Electroanal. Chem., I I (Ig66) 65.

8 B. Breyer and S. Hacobian, Australian J. Sci. Res, A 5 (1952) 500.

9 M. Proskurnin and A. Frumkin, Trans. Faraday Soc., 31 (I935) IIo.

IO J. W. HAYES AND H. H. BAUER, $J$. Electroanal. Chem., 3 (I962) 336.

I $\mathrm{H}$. H BAUER AND D. C. S. Foo, unpublished observations.

I2 B. Breyer, Australian $J$. Sci.. I6 (I953) Iog.

I3 H. H. Bauer, T. Biegler and D. C. S. Foo, Bull. Chem. Soc Japan., 36 (1963) I70o.

I 4 H. MAisuda, $Z$. Elektrochem., 6 I (I957) 489 .

I5 H. H. Bauer AND D. C. S. Foo, Azstralian $J$-Chem., I7 (1964) 510.

I6 H. A. LAItinen and J. E. B. Randles, Trans. Faraday Soc., 5 I (I955) 54.

I7 H. MAtSUdA, $J$. Phys. Chem., 64 (1960) 339.

I8 H. H. Bauer, $J$. Electroanal Chem, I2 (1966) 64. 